\title{
Do monogenic inborn errors of immunity cause susceptibility to severe COVID-19?
}

\author{
Chris Cotsapas, ${ }^{1,2}$ Janna Saarela, ${ }^{3}$ Jocelyn R. Farmer, ${ }^{4}$ Vinod Scaria, ${ }^{5}$ and Roshini S. Abraham ${ }^{6,7}$ \\ ${ }^{1}$ Yale University School of Medicine, New Haven, Connecticut, USA. ${ }^{2}$ The Broad Institute, Cambridge, Massachusetts, USA, ${ }^{3}$ Center for Molecular Medicine, University of Oslo, Oslo, Norway. ${ }^{4}$ Massachusetts \\ General Hospital, Boston, Massachusetts, USA. ${ }^{5} \mathrm{CSIR}$ Institute of Genomics and Integrative Biology, New Delhi, India. ${ }^{6}$ Department of Pathology and Laboratory Medicine, Nationwide Children's Hospital, \\ Columbus, Ohio, USA. ${ }^{7}$ The Ohio State University Wexner College of Medicine, Columbus, Ohio, USA.
}

\begin{abstract}
The SARS-CoV-2 virus, which causes COVID-19, has been associated globally with substantial morbidity and mortality. Numerous reports over the past year have described the clinical and immunological profiles of COVID-19 patients, and while some trends have emerged for risk stratification, they do not provide a complete picture. Therefore, efforts are ongoing to identify genetic susceptibility factors of severe disease. In this issue of the $J C I$, Povysil et al. performed a large, multiple-country study, sequencing genomes from patients with mild and severe COVID-19, along with population controls. Contrary to previous reports, the authors observed no enrichment of predicted loss-of-function variants in genes in the type I interferon pathway, which might predispose to severe disease. These studies suggest that more evidence is needed to substantiate the hypothesis for a genetic immune predisposition to severe COVID-19, and highlights the importance of considering experimental design when implicating a monogenic basis for severe disease.
\end{abstract}

\section{Identifying risk predictors remains a pressing need}

It has been a little over a year since the novel coronavirus infection caused by SARS-CoV-2 was classified as a pandemic by the World Health Organization (WHO), and since then we have seen a dazzling array of studies on all aspects of the virus, its manifestations, and impact on human lives. With over 159 million people infected globally and over 3 million deaths, it has become imperative to identify major risk factors predisposing to infection, disease severity, or mortality. Many studies indicate that severe outcomes are associated with a cytokine release syndrome (1), and that age, several comorbidities, and male sex are associated with higher mortality $(2,3)$. However, these factors do not com- pletely explain the risk of severe outcomes after infection, and identifying other risk predictors remains a pressing need.

One intuitively attractive hypothesis is that an intrinsic immune deficiency might increase the risk of both infection and more severe consequences of infection. Pathogenic variants in over 450 genes have been reported to cause single-gene inborn errors of immunity (IEIs) $(4,5)$, and it is tempting to speculate that the IEI population would be at particular risk. Individuals with variants in almost all the categories of IEIs in the 2019 International Union of Immunological Societies (IUIS) classification of IEIs (4) have been reported to have SARSCoV-2 infection, including those with phenocopies of disease (where the disorder is caused by somatic variants or autoanti-

\section{Related Article: https://doi.org/10.1172/JCI147834}

Conflict of interest: JS holds an investigator-initiated grant from Sanofi Genzyme, and JRF holds investigator-initiated research grants from Bristol Myers Squibb and X4 Pharmaceuticals.

bodies against immunologically relevant proteins, and mimics the phenotype of a genetic defect). The Iranian registry of IEIs reported infections in 19 out of 2,754 assessed patients, which is an incidence 1.23-fold higher than that in the total Iranian population (6). In a retrospective study of SARS-CoV-2-infected IEI patients, 33 of 94 (35\%) had severe outcomes (7). Nine patients died of the infection, indicating a mortality rate of approximately $10 \%$. The age of the deceased patients ranged from infancy to over 75 years, suggesting that predisposition to mortality was based on clinical status at the time of infection, and other comorbidities rather than a specific underlying genetic defect.

Another study of 987 patients with COVID-19 pneumonia found that 101 had autoantibodies against a variety of type I interferons (IFNs), including IFN- $\omega$ and IFN- $\alpha$, or both, and that these autoantibodies were absent in patients with mild or asymptomatic disease, and noted in only a minority of healthy controls (8). In a different study (age range, $8-48$ years; 9 male, 13 female) assessing patients with a specific IEI, autoimmune polyendocrine syndrome type 1 (APS-1), it was observed that of 21 patients tested for type I IFN autoantibodies, all had high titers of neutralizing antibodies against IFN- $\alpha$ and/or IFN- $\omega$, and one patient also had autoantibodies against IFN- $\beta$ (9). Approximately two-thirds of these patients (68\%) had severe COVID-19, and $86 \%$ were hospitalized. These autoantibodies were present before the pandemic, and were thus postulated to predispose to high risk of developing severe SARS-CoV-2 infection. In contrast, Meisel et al. in this issue of the JCI explored a smaller number of APS-1 patients $(n=4$, age $<26$ years, female) who also had preexisting high titers of neutralizing autoantibodies against type I IFNs (IFN- $\alpha$ and IFN- $\omega$ ) and failed to show any predisposition to severe COVID-19; all 
A

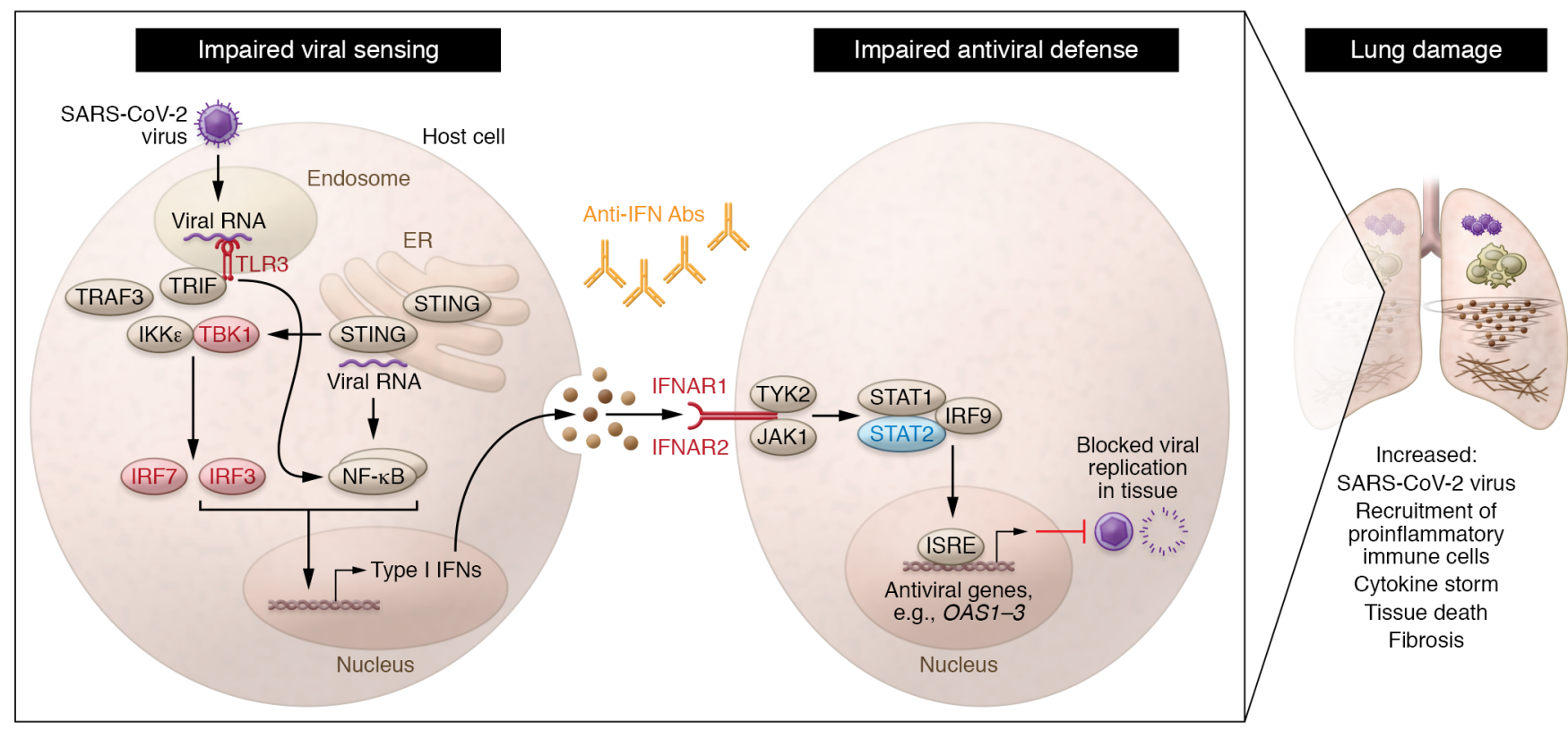

B
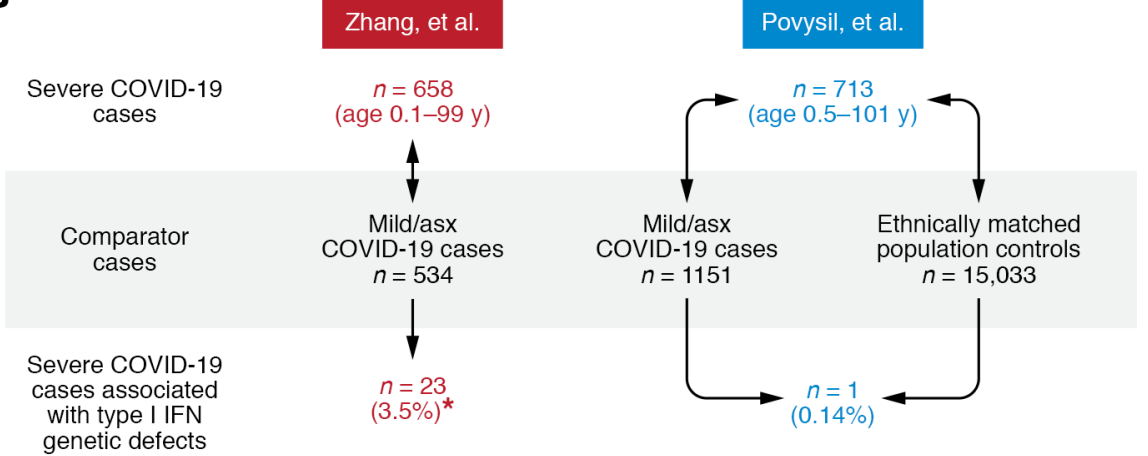

Heterozygous variants: $n=19$

Homozygous variants: $n=4$

Heterozygous variants: $n=1$

Homozygous variants: $n=0$

Figure 1. Defects in innate antiviral signaling linked with increased SARS-Cov-2 disease severity. (A) The type I IFNs are crucial to innate immune responses, and individuals with IEI may carry higher risk for severe COVID-19. Inherited defects associated with SARS-CoV-2 pathogenicity in the Zhang et al. study (13) (red) contrasts with the findings from Povysil et al. (14) (blue). However, other studies also show that acquired, male-predominant production of anti-IFN antibodies increases SARS-CoV-2 pathogenicity (yellow). More evidence is needed to establish a genetic immune predisposition to severe COVID-19. (B) Experimental design differences in the Zhang et al. and Povysil et al. studies include sample size and comparator groups. ${ }^{*} P<0.05$ versus comparator cases. When implicating a monogenic basis for severe disease, best practices involve increasing the sample size, assessing variation across all genes as opposed to a subset, and distinguishing association from cause. Asx, asymptomatic; ER, endoplasmic reticulum; IFNAR, interferon- $\alpha / \beta$ receptor; ISRE, interferon-sensitive response element; IRF, interferon regulatory factor; IKK, IKB kinase; Jak, Janus kinase; NF, nuclear factor; STING, stimulator of interferon genes; SARS, severe acute respiratory syndrome; STAT, signal transducer and activator of transcription; TBK, TANK-binding kinase; TLR, Toll-like receptor; TRAF, TNF receptor-associated factor; TRIF, TIR-domain-containing adapter-inducing interferon- $\beta$; Tyk, tyrosine kinase; $y$, years.

patients had mild disease (10). The type I IFNs are crucial to innate immune responses, as are TLR3, -7 , and -8 , which act as viral sensors. In a study of 50 COVID-19 patients with variable disease severity, those with severe and life-threatening illness had an impaired type I IFN response with a hyperinflammatory component (11). Thus, several strands of evidence support the notion that individuals with immune deficiencies have a higher risk of severe
SARS-CoV-2 infection outcomes. Potential ascertainment biases - on the one hand, these patients are followed more closely, and are consequently more likely to have an infection diagnosed; on the other, they tend to take more precautions than average - make definitive conclusions difficult. The presence of anti-IFN antibodies suggests that at least some patients with severe SARS-CoV-2 infection have defective antiviral signaling, either due to an abnormality in the response to IFN or in IFN production (Figure 1 and ref. 12).

In an orthogonal approach, Zhang et al. performed a genetic study of 659 individuals with life-threatening COVID-19 pneumonia. The authors suggested that IEIs mediate severe SARS-CoV-2 infection outcomes in at least a subset of patients without prior IEI-associated symptoms (13). They argued that an overrepresentation of rare, protein-alter- 
ing genetic variants in 13 genes related to type I IFN on which they had focused, some of which showed variable activity in in vitro biochemical assays, indicated previously undiagnosed IEIs, and thus explained a small proportion of the severe cases in their cohort. In this issue of the JCI, Povysil et al. (14), looking at a substantially larger cohort, found no evidence, in severe cases, of enrichment of rare, protein-altering variants in the genes reported by Zhang et al. In fact, Povysil and colleagues identified one of the initially reported variants (IRF7, p.Gln198*) in three of their controls, and further identified only one loss-of-function (LOF) variant (STAT2, p.Arg330*) in their severe COVID-19 group. Here, we try to reconcile these findings to assess the genetic evidence, and determine whether individuals with IEIs are at greater risk of severe SARS-CoV-2 infection outcomes.

\section{Rare variants in type I IFN genes and severe COVID-19}

Although Zhang et al. (13) sequenced the entire coding portion (exome) of the genome in 295 individuals, and the whole genome in 364 individuals with severe disease, they focused on assessing whether rare, potentially deleterious variants were enriched in 13 genes previously implicated in severe viral infections. They looked for rare variants (at a minor allele frequency of less than $0.1 \%$ ), considering those which changed the sequence of the protein product (missense); or were in-frame insertions or deletions, which added or removed one or more amino acids in an otherwise intact protein; or were predicted LOF (pLOF). They found that 113 of 659 patients with severe COVID-19 were heterozygous for such variants across 12 of the 13 genes they studied, and another four patients were homozygous for such variants in two of those genes. Nine of these 118 variants were pLOF, with the remaining 109 being missense or in-frame indels. In the 534 controls, they found only one pLOF variant in the 13 genes studied. They did not report the number of missense or in-frame indels in the controls. When considering only pLOF variants, they reported enrichment in severe cases compared with mild $(P=0.01$; odds ratio $[\mathrm{OR}]=8.28 ; 95 \%$ confidence interval $[\mathrm{CI}]=1.04$ to 65.64 , under an autosomal dominant model). Zhang et al. (13) then demonstrated that 24 of the 118 variants present in 23 patients, including the nine pLOF, have an effect in in vitro biochemical assays, suggesting altered protein function. They conclude that the carriers of these variants have cryptic IEIs, and that these conditions underlie their severe outcomes. Thus, they suggest that 3.5\% of severe cases are caused by previously undiagnosed IEIs in the population.

In this issue of the JCI, Povysil et al. (14) attempt to replicate the findings of Zhang et al. The authors sequenced 713 patients with severe COVID symptoms, 1,151 with mild disease, and 15,033 population controls (Figure 1). In this substantially larger cohort, they found only one rare $\mathrm{pLOF}$ variant in one individual across the 13 genes postulated by Zhang et al. in their severe disease group, and 23 of the controls, but there was no enrichment of pLOFs in severe cases relative to mild cases. They also did not observe an enrichment relative to matched population controls, which would suggest that variation in these genes is unrelated to susceptibility to infection or severity of outcome. Furthermore, they did not observe an enrichment of rare missense or in-frame indels in these genes. Of note, Zhang et al. have studied such variants found in cases, but not in controls, and do not report how many such variants they found in controls. Therefore, as Povysil et al. note, there is no way of assessing whether these variants are enriched in the severe cases of COVID-19 studied by Zhang et al.

\section{Interpretation and conclusions}

In essence, Zhang et al. make two claims: (a) patients with severe COVID-19 outcomes have a higher frequency of rare pLOF variants in 13 genes, compared with patients with mild infection; and (b) missense, in-frame insertion/deletion, and pLOF variants with variably impaired biochemical activity cause severe SARSCoV-2 infection outcomes in carriers, and effectively constitute previously undiagnosed IEIs. Povysil et al. (14) find no evidence in support of the first claim, but do not directly test the second claim.

Zhang et al. show enrichment in severe cases only for pLOF variants but conclude that all variants with varying degrees of biochemical activity are causal. However, the authors do not present evidence that all classes of rare variants are enriched, nor do they assay missense variants identified in mild cases for biochemical activity to show that this class is enriched in severe cases. Second, the in vitro reporter assays used are based on transfection, and thus tend to mimic the homozygous state, whereas 21 of 23 putative IEI patients are heterozygous for the variant they carry. Further, Zhang et al. assume that these 21 heterozygous alleles must act in an autosomal dominant manner, even though the majority of the 12 autosomal genes they focused on have only been reported with autosomal recessive disease. By making these assumptions, and by considering a single carrier of each variant sufficient proof of causality, Zhang et al. (13) arrive at the conclusion that $3.5 \%$ of severe cases are caused by IEIs.

How then, are we to interpret these claims and the evidence that supports them? And what, if any, relevance do the data of Povysil et al. have for the second claim made by Zhang et al.?

Firstly, it is essential to have a large sample size with appropriate numbers of population-matched controls to eliminate bias introduced from assessing specific populations.

Secondly, it is critical to assess variation across all genes in an unbiased way, rather than preselecting genes representing an a priori hypothesis. While this candidate-gene approach was widely employed in the past, it has largely failed, with most reported candidate-gene studies not replicable in new cohorts $(15,16)$, so it is perhaps unsurprising that Povysil et al. were unable to replicate the associations observed by Zhang et al. This preselection bias was the major impetus for genome-wide study designs (15), which have uncovered roles for thousands of previously unsuspected genes across many immune-relevant traits, and in traits involving every other organ system.

Thirdly - and somewhat counterintuitively - biochemical activity is insufficient in itself to declare that a variant is causal for a trait. Causality can only be demonstrated with evidence that variants are statistically enriched in different groups of patients. Rare variants are, paradoxically, quite common; gnomAD lists almost 450,000 pLOF variants dis- 
covered across over 125,000 individuals, with approximately $40 \%$ of these variants being observed in only one individual (17). A large fraction of these variants is likely to associate with varying degrees of biochemical activity, but that does not necessarily indicate that they cause physiological phenotypes. Although the American College of Medical Genetics (18) and the Association of Molecular Pathology (19) have guidelines for interpreting sequence variants, consensus from scientists and clinicians performing population genetics studies are necessary to ensure conformity remains for both study design and classification.

In summary, there is currently no convincing evidence that individuals with monogenic immune disorders are at increased risk of severe COVID-19 outcomes. The hypothesis remains viable, but more compelling evidence will be needed to substantiate it. Other susceptibility factors include phenocopies of monogenic disease, such as IFN- $\alpha-$ and IFN- $\omega$-neutralizing autoantibodies, with a 15.8-fold male-to-female predominance $(8,20)$. Therefore, COVID-19 infection clinical course is likely to depend on a variety of risk factors, including age, sex, clinical status, immunology, and genetics.

Address correspondence to: Roshini S. Abraham, Department of Pathology and Laboratory Medicine, Nationwide Chil- dren's Hospital, Columbus, Ohio 43205, USA. Phone: 614.722.5329; Email: Roshini. Abraham@nationwidechildrens.org.

1. Carvalho T, et al. The first 12 months of COVID19: a timeline of immunological insights. Nat Rev Immunol. 2021;21(4):245-256.

2. Scully EP, et al. Considering how biological sex impacts immune responses and COVID-19 outcomes. Nat Rev Immunol. 2020;20(7):442-447.

3. Takahashi $\mathrm{T}$, et al. Sex differences in immune responses that underlie COVID-19 disease outcomes. Nature. 2020;588(7837):315-320.

4. Tangye SG, et al. Human inborn errors of immunity: 2019 update on the classification from the International Union of Immunological Societies Expert Committee. J Clin Immunol. 2020;40(1):24-64.

5. Tangye SG, et al. The ever-increasing array of novel inborn errors of immunity: an interim update by the IUIS Committee. J Clin Immunol. 2021;41(3):666-679.

6. Delavari S, et al. Impact of SARS-CoV-2 pandemic on patients with primary immunodeficiency. J Clin Immunol. 2021;41(2):345-355.

7. Meyts I, et al. Coronavirus disease 2019 in patients with inborn errors of immunity: an international study. J Allergy Clin Immunol. 2021;147(2):520-531

8. Bastard P, et al. Autoantibodies against type I IFNs in patients with life-threatening COVID19. Science. 2020;370(6515):eabd4585.

9. Bastard P, et al. Preexisting autoantibodies to type I IFNs underlie critical COVID-19 pneumonia in patients with APS-1. J Exp Med. 2021;218(7):e20210554.

10. Meisel C, et al. Mild COVID-19 despite autoantibodies to type I IFNs in autoimmune-polyendocrine-syndrome type 1 (APS-1). JClin Invest. 2021;131(14):e150867.
11. Hadjadj J, et al. Impaired type I interferon activity and inflammatory responses in severe COVID-19 patients. Science. 2020;369(6504):718-724.

12. Meffre E, Iwasaki A. Interferon deficiency can lead to severe COVID. Nature. 2020;587(7834):374-376.

13. Zhang Q, et al. Inborn errors of type I IFN immunity in patients with life-threatening COVID-19. Science. 2020;370(6515): eabd4570.

14. Povysil G, et al. Rare loss-of-function variants in type I IFN immunity genes are not associated with severe COVID-19. JClin Invest. 2021;131(14):e147834.

15. Border R, et al. No support for historical candidate gene or candidate gene-by-interaction hypotheses for major depression across multiple large samples. Am J Psychiatry. 2019;176(5):376-387.

16. Studies N-NWGoRiA, et al. Replicating genotype-phenotype associations. Nature. 2007;447(7145):655-660.

17. Karczewski KJ, et al. The mutational constraint spectrum quantified from variation in 141,456 humans. Nature. 2020;581(7809):434-443.

18. Richards S, et al. Standards and guidelines for the interpretation of sequence variants: a joint consensus recommendation of the American College of Medical Genetics and Genomics and the Association for Molecular Pathology. Genet Med. 2015;17(5):405-424.

19. Li MM, et al. Standards and guidelines for the interpretation and reporting of sequence variants in cancer: a joint consensus recommendation of the Association for Molecular Pathology, American Society of Clinical Oncology, and College of American Pathologists. J Mol Diagn. 2017;19(1):4-23

20. Koning R, et al. Autoantibodies against type I interferons are associated with multi-organ failure in COVID-19 patients [published online April 9, 2021.]. Intensive Care Med. https://doi.org/10.1007/ s00134-021-06392-4 